\title{
«Brazo y cerebro»: Las dinámicas de inclusión- exclusión en torno a la profesión médica y el anarcosindicalismo español en el primer tercio del siglo XX
}

\section{Jorge Molero-Mesa $(*)$ e Isabel Jiménez-Lucena $(* *)$}

$\left(^{*}\right)$ Centre d'Història de la Ciència. Unitat d'Història de la Medicina. Facultat de Medicina, Universitat Autònoma de Barcelona.

Jorge.Molero@uab.cat

${ }^{* *}$ Unidad de Historia de la Medicina. Facultad de Medicina. Universidad de Málaga. isajimenez@uma.es

Dynamis

[0211-9536] 2013; $33(1): 19-41$
Fecha de recepción: 30 de junio de 2012

Fecha de aceptación: 15 de noviembre de 2012

SUMARIO: 1.- Inclusión y exclusión de los técnicos e intelectuales en el anarcosindicalismo. 2.- - Los Sindicatos Únicos de Sanidad de la Confederación Nacional del Trabajo. 3.-Inclusiónexclusión de los médicos en el anarcosindicalismo. Estrategias, resistencias y limitaciones. 4.-Epílogo.

RESUMEN: El objeto del presente trabajo es analizar la dinámica que se estableció dentro del anarcosindicalismo español entre los trabajadores manuales y los profesionales médicos que comulgaban con la ideología anarquista. La incorporación de técnicos e intelectuales al movimiento obrero fue una característica generalizada en el mundo occidental pero mientras que las organizaciones socialistas dejaban en manos de los médicos la dirección de todas sus políticas sanitarias, los sindicatos anarquistas no aceptaron estos principios tecnocráticos pues no consideraban al intelectual como el más indicado para tomar decisiones que concernían a toda la colectividad. En este contexto, podemos observar como los profesionales de la medicina desarrollaron distintas estrategias para ser aceptados por los militantes anarcosindicalistas, los cuales, a su vez mostraban distintos niveles de aceptación según las distintas corrientes de pensamiento existentes en el sindicato.

PALABRAS CLAVE: anarcosindicalismo, medicina y clase obrera, España, siglo XX, inclusión/ exclusión, obreros intelectuales, ciencia e ideología, tecnocracia.

KEY WORDS: anarcho-syndicalism, medicine and the working class, Spain, 20th century, inclusion/exclusion, intellectual workers, science and ideology, technocracy. 
«Natura no distingue de sabios e ignorantes, de refinados y zafios. Todos, igualmente, animales que comen y defecan. El desarrollo intelectual y afectivo puede constituir una ventaja personal y derivar en provecho común, nunca fundar un privilegio sobre los demás»

Ricardo Mella. El cerebro y el brazo. Acción Libertaria. 1913; 8: 7.

\section{Inclusión y exclusión de los técnicos e intelectuales en el anarcosin- dicalismo (*)}

La consolidación del modelo anarcosindicalista en los primeros años del siglo XX pasaba por garantizar la independencia de los sindicatos obreros en su estrategia apolítica y de acción directa contra el capital. En este periodo, en el que se estaba configurando una clase de sindicalismo que acabaría imponiéndose en los medios libertarios españoles, se evitó que los intelectuales de cualquier signo político e ideológico pudieran imponer una determinada línea de actuación alejada de los intereses de los trabajadores manuales. De esta forma, en el congreso en el que se constituyó la Confederación Regional de Sociedades de Resistencia-Solidaridad Obrera celebrado en Barcelona en septiembre de 1908, incluyó, entre los estatutos de la Confederación, un artículo que admitía la cooperación de los «llamados intelectuales» en la Confederación pero «sin intervención ninguna en su régimen administrativo y directivo» ${ }^{1}$.

Esta línea de pensamiento era la consecuencia, como se confirmaría en el congreso fundacional de la Confederación Nacional del Trabajo (CNT) en 1910, de una especial interpretación del axioma procedente de la Primera internacional que asumía que «la emancipación de los trabajadores ha de ser obra de los trabajadores mismos» y que conformaría uno de los

(*) Esta investigación forma parte de los proyectos HAR2009-13389-C03-01 y HAR2009-13389-C03-03 financiados por el Ministerio de Economía y Competitividad.

1. Bar, Antonio. La CNT en los años rojos: del sindicalismo revolucionario al anarcosindicalismo, 1910-1926. Barcelona: Akal; 1981, p. 128-129. En el primer manifiesto de Solidaridad Obrera, en 1907, se pedía expresamente la afiliación de los intelectuales. Este giro radical puede entenderse por el menor peso de los socialistas en los sindicatos y por la política populista del lerrouxismo que apartó a muchos obreros catalanes del sindicalismo revolucionario. Sobre las diferencias con los socialistas en esta cuestión puede verse Jiménez-Lucena, Isabel. Cambio político y alternativas sanitarias: El debate sanitario durante la Segunda República. Málaga: Universidad de Málaga; 1995; Jiménez-Lucena, Isabel. La cuestión del regeneracionismo sanitario y su debate durante la Segunda República: elementos de clase e ideología. Dynamis. 1998; 18: 285-314. 
fundamentos del sindicalismo revolucionario. El dictamen al tema octavo de este congreso, consideraba que, por sus privilegios, los intelectuales actuaban consolidando el régimen capitalista «y aún procuran servirse de los [obreros] manuales para esos encumbramientos que les hacen placentera y grata [la] vida» ${ }^{2}$. La intervención en el debate de José Negre (1875-1939), Secretario General de la CNT hasta 1914, reflejaba la opinión de un amplio sector del anarcosindicalismo que estuvo en contra de la sindicación de los intelectuales:

«pueden considerarse obreros a los intelectuales, pero siempre que vayan al margen de los sindicatos, no estando dentro de los mismos, pues no siendo iguales los intereses no pueden tampoco ir juntos, sin que esto sea repudiarlos, pues podemos aprovechar sus dotes intelectuales para sacar lo que para nosotros puede ser provechoso, pero rechazando siempre su injerencia en nuestros asuntos» ${ }^{3}$.

A pesar de todo, existía un elevado número de trabajadores intelectuales que, apartados orgánicamente de los sindicatos, comulgaban con los ideales anarquistas dentro de los llamados grupos de afinidad o bien colaborando con sociedades culturales de orientación libertaría ${ }^{4}$. La posibilidad de que estas agrupaciones afines pudieran intervenir de un modo directo en los sindicatos fue discutida en el Congreso de la Confederación Regional del Trabajo de Cataluña (en adelante CRTC) celebrado en Barcelona en junio de 1918 (Congreso de Sans). De acuerdo con los fundamentos sindicalistas y manteniendo la postura adoptada en los congresos anteriores, la resolución final negaba cualquier intervención directa de «entidades puramente ideológicas» ya que estas agrupaciones no eran «de profesión u oficio para la resistencia al capital». Su tarea, no obstante, era bienvenida siempre que trabajasen «al margen de los sindicatos en pro de la emancipación de la clase productora ${ }^{5}$.

2. Bar, n. 1, p. 547.

3. Bar, n. 1, p. 183.

4. Navarro Navarro, Javier. A la revolución por la cultura: prácticas culturales y sociabilidad libertarias en el País Valenciano (1931-1939). València: Universitat de València; 2004. Sobre el papel de los intelectuales en el anarquismo no sindicalista: Girón, Álvaro. En la mesa con Darwin. Evolución y revolución en el movimiento libertario en España (1869-1910). Madrid: CSIC; 2005. Díez, Xavier. El anarquismo individualista en España (1923-1938). Girona: Virus ed.; 2007.

5. Bar, n. 1, p. 382. 
Cerrada esta posibilidad, se advirtió acerca de la situación especial en la que se encontraban los maestros que trabajaban en las escuelas racionalistas, la mayoría de ellas creadas por los mismos sindicatos que les negaba la afiliación. En consecuencia, una adición a la resolución anterior reconocía la inclusión en la Confederación catalana de estos profesionales por ser «un elemento necesario para la lucha por la emancipación» ${ }^{6}$. A partir de este momento, podemos observar un cambio radical a favor de la sindicación de los intelectuales que, apoyado desde las páginas de Solidaridad Obrera ${ }^{7}$, culminaría meses después, en octubre de 1919, con la creación del Sindicato Único de Profesiones Liberales, adscrito a la Federación Local de Barcelona.

Este giro estratégico se confirmó a nivel estatal en el segundo Congreso de la CNT celebrado dos meses después en Madrid (diciembre, 1919). El dictamen de una de las ponencias aprobadas proponía, con una clara finalidad postrevolucionaria, sindicar a todos los obreros, organizando «rápidamente los Sindicatos de distribución y los de profesiones técnicas y no manuales» ${ }^{8}$.

Podemos observar en esta estrategia un proceso de inclusión-excluyente ${ }^{9}$ que adaptaba la participación de los obreros intelectuales a las necesidades que en ese momento tenía el anarcosindicalismo. La solución encontrada para la sindicación de los técnicos y otros intelectuales significaba una contradicción con la reforma orgánica que se acababa de realizar en el Congreso de Sans y que luego se extendería a toda la CNT y que consistió en agrupar los diferentes sindicatos de oficios en sindicatos de ramo o de industria. Con la agrupación de los intelectuales en un sindicato único se evitaba el peligro, siempre temido, del dirigismo que podían acabar realizando sobre los trabajadores manuales si cada intelectual ingresaba en el sindicato correspondiente a su ramo. Además, desde el congreso fundacional de la CNT, se contemplaba la posibilidad de expulsar de la Confederación

6. Bar, n. 1, p. 383.

7. Trujillo, M. Orientaciones para el movimiento obrero en la actualidad. Solidaridad Obrera. 5 Ene 1919. El éxito de la Revolución soviética y la intransigencia exacerbada de la patronal, favoreció la orientación de la CNT hacia soluciones revolucionarias a corto plazo lo que supondría contar en el sindicato con técnicos capaces de ayudar a mantener el posible régimen libertario. Bar, n. 1, p. 546.

8. Bar, n. 1, p. 546.

9. Bohn, Cornelia. Inclusion and exclusion: Theories and findings. From exclusion from the community to including exclusion. In: Gestrich, A.; Raphael, L.; Uerlings, H., eds. Strangers and poor people. Changing patterns of inclusion and exclusion in Europe and the Mediterranean world from Classical antiquity to the present day. Frankfurt: Peter Lang; 2009, p. 35-53. 
a los obreros que «por su trabajo» pudieran perjudicar directamente a la organización sindical. Consecuentemente, cuando se constituyó en Barcelona el Sindicato de Profesiones Liberales se impidió la afiliación de los redactores del periódico La Publicidad, del que había sido recientemente despedido Andreu Nin (1892-1937), así como a los miembros del somatén, bestia negra del anarcosindicalismo junto con los pistoleros de los sindicatos libres en esos momentos ${ }^{10}$.

Tras el paréntesis que supuso para el anarcosindicalismo la Dictadura de Primo de Rivera, el debate sobre los intelectuales volvió a la CNT con especial intensidad a la vez que se legalizaba ésta en la primavera de 1930. Durante la dictadura, algunos intelectuales siguieron colaborando con las publicaciones culturales de orientación anarquista que lograron mantenerse y pocos en la militancia subversiva de la ilegalizada $\mathrm{CNT}^{11}$. A partir de la legalización de la Confederación, en 1930, el número de trabajadores intelectuales que se incorporaron a la misma fue aumentando muy lentamente pero no de forma significativa. No obstante, a comienzos de junio de 1931, se creó el Sindicato de Obreros Intelectuales y Profesiones Liberales de Barcelona con la finalidad inmediata de poder participar en el congreso extraordinario que la CNT organizaría en Madrid pocos días después. En este congreso, efectivamente, se confirmó la inclusión de los intelectuales y se reconoció, por primera vez, que éstos no solo eran necesarios para hacer funcionar una futura sociedad libertaria sino que, además, había que contar con ellos para preparar la revolución ${ }^{12}$.

Este sindicato, denominado en el lenguaje de la Confederación como «intelectuales», entusiasmado por los resultados del congreso extraordinario, publicó varios artículos en Solidaridad Obrera que contribuyeron al aumento de las tensiones entre las distintas facciones que componían la CNT. Junto a una carta poco afortunada en la que pedían mayor protagonismo en el periódico confederal por la impericia de los obreros manuales en la redacción de $\operatorname{textos}^{13}$, manifestaron que aspiraban a captar a «toda

10. Las profesiones liberales. La Correspondencia de España. 16 Oct 1919.

11. Christie, Stuart. Nosotros los anarquistas: Un estudio de la Federación Anarquista Ibérica (FAl). Valencia: Universitat de València; 2010, p. 65.

12. Ponencia sobre la posición de la CNT frente a las Cortes Constituyentes. Solidaridad Obrera. 5 Jul 1931. Sobre las funciones que desempeñarían los técnicos en los sindicatos para preparar la revolución, véase: Los técnicos en nuestra organización. Solidaridad Obrera. 4 Jul 1931.

13. Tarrega, Enrique. A los delegados del pleno regional. Los intelectuales piden actuar. Solidaridad Obrera. 13 Oct 1931. El revuelo fue tal que poco después la Junta del Sindicato 
esa nube de intelectuales que mariposean alrededor de la CNT que para tener derecho a manifestar su simpatía por nuestra organización necesita poseer nuestro carnet». Todavía iban más allá pues ofrecían la afiliación a todos los intelectuales independientemente de su residencia «al ser este el único Sindicato del ramo existente en España y tener, por lo tanto, circunstancialmente, carácter nacional» ${ }^{14}$.

El temor a que el Sindicato de Intelectuales se convirtiera en una puerta sin el control de los obreros manuales dadas las facilidades que concedía la autogestión organizativa, hizo que los sectores radicales se decantaran por la llamada «síntesis de clase» ${ }^{15}$ y defendieran una nueva estrategia de inclusión-excluyente consistente ahora en integrar a los intelectuales y técnicos en sus respectivos sindicatos de industria.

La Federación Nacional de Sindicatos Únicos de las Industrias del Gas, Agua y Electricidad publicó inmediatamente un manifiesto en el que pedían la disolución del Sindicato de Intelectuales y que se integraran en sus respectivos sindicatos de industria para evitar a tiempo que «puedan llegar a creerse moralmente investidos de una misión pedagógica y encauzadora, y por ende, rectora, de las inquietudes constructivas del cuerpo confederal ${ }^{16}$. La Junta del Sindicato de Intelectuales se defendió afirmando que no vivían «facciosa y antifederalmente» y que, precisamente, en los sindicatos de industria, sí se encontrarían en condiciones propicias "para prevalerse de su superior instrucción e intentar predominar erigiéndose en caudillos o líderes» ${ }^{17}$.

Se temía, como antaño, el «dirigismo» que podrían llegar a desempeñar en la CNT los anarquistas específicos, pero también que los grupos de intelectuales derivaran en organizaciones políticas radicales de izquierda o incluso en grupos fascistas dónde muchos intelectuales con inquietudes políticas habían recalado. Recientemente, había sido expulsado de la Fede-

de Intelectuales tuvo que desmentir la autoría de la carta. La Junta. Aclaración. Solidaridad Obrera. 23 Oct 1931.

14. El Comité. Los intelectuales responden. Solidaridad Obrera. 14 Jun 1932.

15. Así era denominada la unión entre los trabajadores manuales e intelectuales para aumentar la eficacia de lucha frente al capital. También defendían el equilibrio que debía existir en cada persona entre la actividad manual e intelectual. Ambos objetivos fueron la razón de ser de la revista Brazo y cerebro, fundada en 1932 como órgano de los sindicatos de la comarca de Tarrasa.

16. Los intelectuales y la CNT. Solidaridad Obrera. 29 Jun 1932.

17. La Junta. Los intelectuales y la CNT. Solidaridad Obrera. 17 Jul 1932. 
ración madrileña un grupo de intelectuales del Sindicato de Sanidad por haber creado una organización paralela denominada «Técnica y Trabajo» (TECTRA) de tintes fascistas y rituales masónicos ${ }^{18}$. Por otro lado, en el Sindicato de Intelectuales de Barcelona militaban conocidos comunistas que pertenecían al Bloque Obrero y Campesino y al partido Extrema Izquierda Federal, organizaciones cuya estrategia pasaba por atraerse a las masas anarcosindicalistas ${ }^{19}$.

La controversia no llegó a resolverse en el periodo estudiado ${ }^{20}$. Fruto del rechazo obrero, los intelectuales desplegaron una serie de estrategias tendentes a ser aceptados de forma definitiva en el anarcosindicalismo y que será el objeto de los apartados siguientes tomando a los profesionales de la medicina como modelo. Permanentemente cuestionados, los intelectuales representaron siempre una minoría con relación a los trabajadores manuales. Dada la estructura interna de la CNT, es complicado conocer de forma exacta el número y procedencia de los intelectuales afiliados a esta central. A pesar de que no existió otro sindicato específico fuera de Barcelona, este podía acoger a intelectuales de otras localidades. También, los sindicatos de obreros manuales podían incluir entre sus filas a este tipo de trabajadores por lo que habrá que esperar a estudios locales que nos informen sobre esta cuestión ${ }^{21}$.

18. Federación Local de Sindicatos Únicos de Madrid. La Libertad. 6 Feb 1932. Urrutia, Federico de. Las divergencias entra la FAl y la CNT ¿Qué es la «Tectra»? Heraldo de Madrid. 23 Feb 1932.

19. A lo largo de 1932 se expulsaron del Sindicato de Intelectuales al maestro Víctor Colomer i Nadal (1896-1960) y al militar José García Miranda (n. 1897) ambos «candidatos del Bloque Obrero y Campesino» y al médico Diego Ruiz Rodríguez (1881-1959) por ser «candidato de la Extrema Izquierda Federal». Sindicato de Obreros Intelectuales. Solidaridad Obrera. 16 Nov 1932.

20. Sobre este tema durante la Guerra civil véase: Fernández Soria, Juan Manuel. Cultura y Libertad. La educación en las Juventudes Libertarias (1936-1939). Valencia: Universidad de Valencia; 1996, p. 189-205.

21. El Sindicato de Profesiones Liberales de Barcelona tenía en 1919, un total de 150 miembros lo que significaba tan sólo un 0,18\% del total de los afiliados de la CRTC. En abril de 1931, ya como Sindicato de Obreros Intelectuales y Profesiones Liberales, se elevó el número a 200 pero, con la fuerte subida de militancia obrera en ese año, el porcentaje fue del 0,07\%. En abril de 1932, los 300 afiliados suponían un 0,13\% del total de afiliados. En marzo de 1933, ese porcentaje ascendió al 0,15\% (315 afiliados). En 1936, el número de intelectuales afecto a la CRTC descendió a 230, lo que representaba un 0,17\% del total de afiliados. Para estas cifras, véase: El Congreso Confederal de la Comedia. Revista de Trabajo. 1975; 49-50: 205-506; Vega, Eulalia. Entre revolució i reforma. La CNT a Catalunya (1930-1936). Lleida: Pagès; 2004, p. 140. 


\section{Los Sindicatos Únicos de Sanidad de la Confederación Nacional del Trabajo}

La presencia regular de médicos y médicas en los movimientos anarquistas europeos y en general, dentro del movimiento obrero, se ha justificado tradicionalmente por una pretendida «conciencia social» que algunos de estos profesionales adquirieron por haber sido testigos directos y cercanos de los estragos que la revolución industrial y el auge del capitalismo estaban ejerciendo sobre la salud de los trabajadores ${ }^{22}$. Sin embargo, la «clase médica» en general no estaba mejor considerada que el resto de los grupos de técnicos e intelectuales que, por un motivo u otro, se acercaban a las filas anarcosindicalistas. La aceptación, siempre discutida, pasaba por la actuación del médico en la organización como activista revolucionario antes que por su condición de técnico lo que no le suponía ninguna ventaja añadida a la de un obrero manual dentro del sindicato. La condición de técnicos militantes tampoco suponía a los médicos quedar al margen de la censura ideológica ejercida por las publicaciones anarcosindicalistas ${ }^{23}$.

Junto al rechazo de los principios tecnocráticos, las peculiaridades de la lucha anarcosindicalista basada en la acción directa y el apoliticismo, dejaba a los profesionales de la medicina sin opciones de liderazgo político y sin oportunidades de utilizar sus conocimientos técnicos para intermediar con el poder, tener influencia social o codearse con las élites del poder. Los defensores de la sindicación de los técnicos pensaban que si alguno de ellos intentaba utilizar la plataforma de la CNT para su «encumbramiento personal», fracasarían estrepitosamente «por la falta de ambiente» y que, además, no faltarían ocasiones "para hacerle ver que había equivocado el camino; por tomar la ruta de la UGT, había tomado la de la $\mathrm{CNT}_{\gg}{ }^{24}$. En efecto, caso muy distinto era el de los médicos socialistas que en los años

22. Martí Boscà, José Vicente. La sanidad libertaria en España. Solidaridad Obrera. [Centenario de la CNT] Feb 2010: 52.

23. En marzo de 1932, la redacción de Solidaridad Obrera, rechazó la publicación de un artículo de Augusto M. Alcrudo (1892-1936) y le advertían: «no podemos seguirte por ese camino». Redacción. Solidaridad Obrera. 15 Mar 1932. Incluso el mismo Isaac Puente (1896-1936) fue objeto de ciertas reprobaciones, véase Jiménez Lucena, Isabel. Asistencia sanitaria de, por y para los trabajadores: sanidad y anarquismo durante la Segunda República. In: Martí, J.V.; Rey, A., eds. Actas del I Simposium Félix Martí lbáñez: medicina, historia e ideología. Valencia: Generalitat Valenciana; 2004, p. 141-159.

24. Urbinaga, Juan de. Hacia la sindicación de los técnicos. Solidaridad Obrera. 13 Oct 1931. 
finales de la Dictadura de Primo de Rivera irrumpieron de forma notable en la Unión General de Trabajadores (UGT) y en el Partido Socialista Obrero Español (PSOE) como responsables de sus respectivas políticas sanitarias ${ }^{25}$. Alcanzaron una gran visibilidad en el parlamento republicano y, en algunos momentos, crearon grupos de presión con otros diputados médicos no socialistas en defensa de su «clase» ${ }^{26}$.

Las luchas corporativas ocupaban en estos años a una mayoría de médicos que aspiraban a mayores cotas de poder dentro del sistema liberal y a la instauración de una tecnocracia médica en el seno del Estado ${ }^{27}$. En este sentido, entre las campañas desarrolladas por diversos sectores de la medicina hegemónica podemos destacar las llevadas a cabo para que se crease en España un Ministerio de Sanidad y la que protagonizaron los médicos titulares para ser funcionarios del Estado ${ }^{28}$. También, y en la misma línea de actuación, podemos señalar las movilizaciones de los médicos que participaban en la campaña antituberculosa de iniciativa privada para que el Estado asumiera todo el protagonismo de la misma y los incorporara a su correspondiente escalafón ${ }^{29}$.

En cualquier caso, la CNT nunca logró atraer a los médicos de forma significativa en el periodo estudiado a pesar de que, a partir de 1930, fueron varios los sindicatos de sanidad que se crearon en algunas capitales de provincia ${ }^{30}$. Para coordinar la labor de los mismos, el Sindicato Único de Sanidad de Madrid convocó un congreso nacional (Madrid, 8 al 15 de noviembre de 1931) para crear una Federación de Sindicatos Únicos de Sanidad unos meses después de que el congreso extraordinario de la CNT

25. Molero-Mesa, Jorge. Clase obrera, Medicina y Estado en la España del Siglo XX. Bases sociopolíticas de las campañas antituberculosas entre 1889 y 1950. In: Castellanos, Jesús et al., eds. La medicina en el siglo XX. Estudios Históricos sobre Medicina, Sociedad y Estado. Málaga: SEHM; 1998, p. 221-228.

26. Jiménez-Lucena, 1998, n. 1.

27. Jiménez-Lucena, Isabel. El Estado como aliado. Los médicos y el proceso de estatalización de los servicios sanitarios en la Segunda República española. Asclepio. 1997; 14: 193-216.

28. Huertas, Rafael. Organización sanitaria y crisis social en España. La discusión sobre el modelo de servicios sanitarios públicos en el primer tercio del siglo XX. Madrid: Fundación de Investigaciones Marxistas; 1994.

29. Molero-Mesa, Jorge. Historia Social de la Tuberculosis en España (1889-1936). Granada: Universidad de Granada; 1989.

30. En el caso catalán podemos señalar también la existencia, desde 1920, del Sindicat de Metges de Catalunya, organización única en Europa que agrupaba a la mayoría de los médicos catalanes de forma corporativa al margen de los colegios oficiales. 
incluyera este nivel en su organigrama. A pesar de que la convocatoria pedía que acudiesen «el mayor número de delegados que los efectivos de cada Sindicato les permitan» sólo asistieron quince, representando a los sindicatos de Madrid, Zaragoza, Álava y Teruel. No se presentó la delegación de Santiago de Compostela, que había anunciado su asistencia ${ }^{31}$, y se recibieron adhesiones de Sevilla y Huelva ${ }^{32}$. El sindicato de Barcelona, el más numeroso en esos momentos, no acudió de forma significativa ${ }^{33}$.

Al congreso extraordinario que la CNT había organizado meses antes, sólo acudieron delegados del Sindicato de Sanidad de Madrid (195 afiliados), Zaragoza (10) y Barcelona (1.460), siendo este último sindicato el único que no incluía a ningún médico entre sus delegados ${ }^{34}$. En el congreso de la CNT de 1936, algunos de estos sindicatos habían desaparecido. Sólo acudieron las delegaciones de Zaragoza (50 afiliados), Madrid (95) y la del Sindicato de Sanidad Marítima de La Coruña $(284)^{35}$. En la convocatoria que hizo el Sindicato de Sanidad de Madrid para su constitución, en octubre de 1930, podemos observar quiénes eran los candidatos a ingresar al sindicato y los motivos por los que tenían que hacerlo:

«Explotado y atropellado el médico por las Compañías de Seguros, por las Sociedades de Asistencia Médica, por el bochornoso caciquismo y hasta por los mismos profesionales que gozan de situación de excepción. Vejado y mal retribuido el practicante. Tratado el enfermero y asistenta de hospital peor que ningún otro obrero. Relegado el farmacéutico a la categoría de vulgar

31. El Sindicato de Santiago de Compostela estaba conformado básicamente en esos momentos por estudiantes de medicina. Martí Boscà, José Vicente. Isaac Puente, el médico que quiso curar al pueblo. In: Fernández de Mendiola, Francisco, ed. Isaac Puente. El médico anarquista. Tafalla: Txalaparta; 2007, p. 113-146.

32. El Sindicato Único de Sanidad de Huelva estaba siendo organizado en esos momentos por algunos practicantes interesados en ingresar en la CNT. Blanca, Benito. Desde Huelva. Los practicantes. Solidaridad Obrera. 7 Jun 1931. Tenemos que tener en cuenta que en algunas poblaciones dónde el número de trabajadores sanitarios no era suficiente podían afiliarse en el Sindicato de Oficios varios o bien en el Sindicato Único de Trabajadores de la localidad.

33. Congreso de Sindicatos Únicos de Sanidad. Solidaridad Obrera. 22 Nov 1931.

34. Assistents al Tercer Congrés Confederal de la CNT. [citado 10 Jun 2012]. Disponible en: http:// www.veuobrera.org/00finest/931assis.htm. Por el Sindicato madrileño acudió Antonio Gascuñana Martín (1907-1933), médico de la Beneficencia Provincial; Nicasio Álvarez de Sotomayor (1900-1936), médico; y Francisco Trigo Domínguez, perito químico; por Zaragoza acudió Augusto Moisés Alcrudo, médico; y por Barcelona Juan Antonio Lorenzo Benito (1878-1938), practicante titulado.

35. Calero, Juan Pablo. Vísperas de la revolución. El congreso de la CNT (1936). Germinal. 2009; (7): 97-132.1 
comerciante. Víctima el auxiliar de farmacia del codicioso comercialismo. Entregado el odontólogo a una lucha fratricida. Eliminado el veterinario del papel sanitario que le corresponde. Sufriendo las consecuencias del desbarajuste profesional los protésicos, desinfectores» ${ }^{36}$.

Sin embargo, los afiliados a los sindicatos de sanidad representados en estos congresos eran mayoritariamente practicantes, «enfermeros» (hombres y mujeres), auxiliares sanitarios de todo tipo y subordinados de los médicos en clínicas y dispensarios, por lo que no podían dejar de alimentar recelos y desconfianza sobre los compañeros de sindicato que provenían de un grupo social en posición de dominación jerárquica. Las sospechas de «dirigismo» que pudieran ejercer los técnicos dentro del sindicato debieron de acrecentarse cuando en el congreso que fundó la Federación de Sindicatos de Sanidad, se crearon dos agrupaciones profesionales dentro de estos sindicatos según los niveles de capacitación del trabajador, distinguiendo entre los profesionales titulados y los no titulados ${ }^{37}$, algo inédito en el seno de la CNT y que, de alguna forma, recordaba a la división jerárquica existente en el sistema sanitario ${ }^{38}$. No en vano, una de las concepciones del anarcosindicalismo era la diferenciación entre capacitación y categoría profesional, siendo esta última una de las causas que más dividía a los trabajadores. Según un artículo de opinión publicado en Solidaridad Obrera en 1931, la categoría profesional era «simplemente, una expresión de la organización capitalista de la producción» dónde «no siempre son los más capaces ni los más decentes los que llevan la marcha del trabajo»; su

36. A los trabajadores del ramo de la sanidad. Solidaridad Obrera. 8 Oct 1930.

37. «Al grupo de profesionales titulados pertenecerán los médicos, farmacéuticos, veterinarios, practicantes de Medicina, comadronas, odontólogos, profesores de cultura física y enfermeros titulados. Al segundo grupo se afiliarán protésicos, practicantes de farmacia, enfermeros no titulados, mozos de hospital, de clínica y de laboratorio o instituto biológico o clínico, camilleros, masajistas, callistas y, por existir un Sindicato de Aseo e Higiene, se respeta el derecho de autodeterminación de los peluqueros». Congreso de Sindicatos Únicos de Sanidad. Solidaridad Obrera. 22 Nov 1931.

38. Detrás de esta separación también se escondía la fuerte lucha profesional que se estaba produciendo entre odontólogos, matronas y practicantes titulados contra toda una serie de nuevos profesionales sanitarios sin titulación a los que acusaban de intrusismo. Todo ello con el trasfondo de las luchas corporativas frecuentes entre los profesionales sanitarios desde mediados del siglo XIX y muy virulentas en esos momentos. Montesinos Vicente, Fernando. Practicantes, matronas y cirujanos dentistas en la España contemporánea (1855-1932). Girona: Universitat de Girona; 2011. 
autoridad colocaba a las «categorías» profesionales al lado del capitalismo contra el resto de los trabajadores ${ }^{39}$.

Después del congreso de noviembre de 1931, los sindicatos de sanidad quedaron sujetos a las luchas intestinas, tanto entre los profesionales sanitarios que los componían como por las tendencias anarcosindicalistas que discrepaban sobre la estrategia a seguir en el camino de la revolución social. La Federación de Sindicatos de Sanidad fracasó, no llegándose a reunir nunca más. Hay que tener en cuenta que los sectores más radicales del anarcosindicalismo se opusieron a la existencia de estos órganos centralizadores a los que consideraban más cercanos al tipo de estrategia marxista en la lucha por el poder que a las tácticas y principios anarquistas en los que la autonomía de los sindicatos locales era fundamental.

En efecto, coincidiendo con la pérdida de influencia del sector reformista dentro de la CNT, los problemas se acrecentaron en los dos principales sindicatos de sanidad. En Madrid, como hemos visto en el apartado anterior, fue expulsado, en febrero de 1932, un grupo de intelectuales del Sindicato de Sanidad, encabezado por los médicos Nicasio Álvarez de Sotomayor y Miguel Palacios Martínez (1895-1979), por crear la agrupación TECTRA al margen del sindicato ${ }^{40}$. La prensa madrileña interpretó estas expulsiones como una consecuencia de las divergencias entra la FAI y la $\mathrm{CNT}^{41}$. Lo cierto es que Nicasio Álvarez acabaría militando de forma activa en las Juventudes de Ofensiva Nacional Sindicalista y colaborando en la creación de la Central Obrera Nacional-Sindicalista ${ }^{42}$. La representación médica en el sindicato madrileño siempre fue muy minoritaria en relación al resto de afiliados como atestigua el médico Juan Morata Cantón (1899-1994), uno de sus fundadores ${ }^{43}$.

El caso de Barcelona reúne unas condiciones especiales debido al fuerte contraste que significaba, por una parte, la fuerza y tradición anarcosindicalista en Cataluña y la presencia de un sindicato corporativista que acogía

39. Las categorías profesionales. Solidaridad Obrera. 28 Jun 1931.

40. Federación, n. 18. Nicasio Álvarez era Secretario de la Federación Local de Sindicatos Únicos de Madrid. Miguel Palacios, era Capitán médico, lo que también pudo influir en la consideración peligrosa de esta agrupación para los intereses de la CNT.

41. Urrutia, n. 18.

42. Rodríguez Arroyo, J. Carlos. Nicasio Álvarez de Sotomayor Gordillo y Aguilar «Ángel Aguilar»: del anarcosindicalismo al nacional-sindicalismo. Revista de Estudios Extremeños. 2010; 56 (3): 1257-1308.

43. Citado por Martí Boscà, n. 31, p. 144. 
a la mayoría de los médicos catalanes. No es casualidad, por tanto, que el primer llamamiento a la creación de un Sindicato de Sanidad adscrito a la $\mathrm{CNT}$ en Barcelona la hiciera un practicante, Juan Antonio Lorenzo Benito, a través de un artículo titulado «La proletarización del médico» ${ }^{44}$. La revista en la que se publicó este artículo, Unión Sanitaria Nacional, era propiedad del médico Alfredo Royo Lloris, y había sido creada en 1928 como pretendido órgano de expresión de los médicos titulares inspectores de sanidad municipal, un grupo caracterizado por sus reivindicaciones gremialistas. Si lo que pretendía esta campaña era atraer a los médicos, no lo consiguió. Según la crónica publicada en Solidaridad Obrera, la asamblea en la que se constituyó el sindicato en diciembre de 1930, contó «con escasa comparecencia» $\mathrm{y}$ «la concurrencia mayor (...) fueron las simpáticas mujeres que (...) alegraron las horas que a su lado pasamos sin sentir». Juan Antonio Benito fue elegido presidente del sindicato y Alfredo Royo, "contador» ${ }^{45}$. En el sindicato sólo pervivió durante algunos meses la sección de enfermeros, enfrentada al régimen de trabajo impuesto a este colectivo en los hospitales de Barcelona ${ }^{46}$. El sindicato se disolvió en septiembre de 1932 con la integración de esta sección en el Sindicato de Productos Químicos ${ }^{47}$, en el que permanecieron hasta después del comienzo de la Guerra Civil cuando se creó de nuevo el Sindicato de Sanidad ${ }^{48}$.

En el fracaso del Sindicato de Sanidad podemos ver la complejidad de las dinámicas de inclusión-exclusión que se estaban desarrollando en el seno del anarcosindicalismo entre los obreros manuales y los intelectuales. Al tradicional recelo y desconfianza que los obreros manuales manifestaron hacia los intelectuales y la tradicional jerarquía profesional que caracterizaba el mundo sanitario, hay que añadir la ideología anarquista individualista de muchos médicos que se acercaban a la CNT convencidos de que era imprescindible contar con las masas obreras que la componían para la

\footnotetext{
44. Lorenzo Benito, J. La proletarización del médico. Unión Sanitaria Nacional, Oct 1930: 2-4.

45. Asamblea del Sindicato de Sanidad e Higiene. Solidaridad Obrera. 11 Dic 1930. El resto de los cargos fueron: Secretario: Nieves Berges y Tesorero: Pedro Sáez López.

46. Un enfermero. Crítica situación de los enfermeros en los hospitales de Barcelona. Solidaridad Obrera. 5 Abr 1931. Monier, Daniel ¿Qué pasa en la Quinta de Salud «La Alianza»? Solidaridad Obrera. 17 Abril 1932. Lo que pasa en el Hospital de San Pablo. Solidaridad Obrera. 18 Ene 1935.

47. Llamamiento a nuestros compañeros de Sanidad. Solidaridad Obrera. 25 Sep 1932.

48. No tenemos noticias de lo ocurrido con el resto de los afiliados pero podemos sospechar, como hemos visto con anterioridad, de la influencia negativa del corporativismo profesional de los practicantes, las matronas y los odontólogos. Montesinos Vicente, n. 38.
} 
construcción de un futuro libertario. Este fenómeno explica la estrategia seguida por los médicos anarquistas, y en general por todos los intelectuales, para ser aceptados plenamente en este movimiento sindical y poder participar en el mismo de forma directa sin permanecer exclusivamente en los ámbitos culturales periféricos del movimiento revolucionario ${ }^{49}$. Los argumentos que ofreció la Junta del Sindicato de Intelectuales para no incorporarse a sus sindicatos de industria, tal como demandaron los sindicalistas manuales, estaban basados precisamente, en el fracaso de los sindicatos de sanidad para atraer a los profesionales de la medicina. Para esta Junta, los intelectuales, se encontraban «descentrados» dentro de los sindicatos de oficios manuales dada su «heterogeneidad [cultural]», sin embargo, en el Sindicato de Intelectuales,

«encontrándose en él reunidas numerosas personas de profesiones varias, pero de educación y cultura semejantes, brota espontáneamente la discusión, y las ideas se pulen y fortalecen, mientras que los que se acercan a nosotros llenos de prejuicios hijos de su anterior actuación aburguesada, encuentran en nuestro Sindicato la adecuada pedagogía para hacer nacer en sus pechos la camaradería más amplia hacia nuestros hermanos manuales» ${ }^{50}$.

La estrategia se completó con la demostración de su comunión con los principios anarquistas basados en el apoliticismo y la acción directa, remitiéndose al ejemplo de «compañeros bastante conocidos en todos los medios sindicales» ${ }^{51}$ para que desechasen «por completo el temor de que nosotros podamos nunca aspirar a preponderar a causa de nuestra intelectualidad».

49. Sobre la labor de los médicos anarquistas en estos ámbitos, véase: Barona, Josep Lluís. Ciencia y revolución en la España de Martí Ibáñez. In: Martí; Rey, eds., n. 23, p. 17-38.

50. La Junta, n. 17.

51. Se refería sin duda a los intelectuales que también actuaban en la FAl y que estaban contribuyendo al desarrollo teórico del anarquismo y a la praxis revolucionaria de la CNT. En nuestro caso, el médico Isaac Puente, además de su militancia activa y su presencia en varios comités revolucionarios, elaboró una extensa obra teórica sobre el anarquismo difundida en la prensa anarcosindicalista que también estaría actuando en las dinámicas de inclusión-exclusión de los médicos en la CNT pero que escapa de los objetivos de este trabajo. Su obra El Comunismo libertario, publicada en 1933, alcanzó en tres años, los cien mil ejemplares y ha sido recientemente reeditada en: Puente, Isaac. El comunismo libertario y otras proclamas insurreccionales y naturistas. Bilbao: Likiniano Elkartea; 2003. Sobre su militancia y aportaciones teóricas al anarquismo véase: Fernández de Mendiola, n. 31, p. 113-146 y Reboredo Olivenza, José Daniel. Teoría política y praxis social de un anarquista vasco: Isaac Puente (1896-1936). Vitoria: ed. del autor; 1995. 
También ofrecieron como prueba «varios casos conocidos por todos» de la expulsión o rectificación de actitudes de intelectuales que se habían acercado al sindicato «creyéndose que vienen a dirigir los movimientos de la Confederación, movidos por la ignorancia o por la ambición congénita» ${ }^{52}$.

El fracaso de los sindicatos de sanidad es un claro exponente de la brecha abierta entre los intelectuales y los obreros manuales en el seno del anarcosindicalismo. Si la mayoría de los médicos no optaba por sindicarse en la CNT por las razones que hemos ido exponiendo anteriormente, los pocos médicos atraídos por las ideas anarquistas preferían ingresar en el Sindicato de Intelectuales al no encontrar una vía de actuación satisfactoria dentro de los sindicatos de sanidad. Además de su posición minoritaria, el carácter liberal de la medicina que practicaban tampoco encajaba con la lucha económica ejercida por los proletarios sanitarios.

Ante esta situación, los médicos anarquistas optaron por una estrategia inclusiva que les permitiera una actuación directa sobre las líneas maestras que debían llevar a la CNT al triunfo revolucionario desde su condición de intelectuales. Independientemente de la plataforma sindical a la que pertenecieran, los profesionales de la medicina se mostraron dispuestos a colaborar con sus conocimientos y ponerlos al servicio de los obreros manuales, como veremos a continuación.

\section{Inclusión-exclusión de los médicos en el anarcosindicalismo. Estra- tegias, resistencias y limitaciones}

Podemos distinguir un primer nivel de actuación de los profesionales médicos en el anarcosindicalismo que consistió en la reformulación de los conocimientos médicos teniendo como principios el igualitarismo libertario y el ambientalismo radical ${ }^{53}$. El conocimiento médico, convenientemente resignificado, podía utilizarse como una herramienta legitimada científicamente contra la medicina oficial y la actuación del médico dentro de ella ${ }^{54}$.

\footnotetext{
52. La Junta, n. 17.

53. Jiménez Lucena, n. 23. Molero-Mesa, Jorge; Jiménez-Lucena, Isabel. «Otra manera de ver las cosas». Microbios, eugenesia y ambientalismo radical en el anarquismo español del siglo XX. In: Miranda, M.; Vallejo, G., eds. Darwinismo social y eugenesia. Derivas de Darwin: cultura y política en clave biológica. Buenos Aires: Siglo XXI; 2010, p. 143-164.

54. Jiménez-Lucena, Isabel; Molero-Mesa, Jorge. Good birth and good living. The (de)medicalizing key to sexual reform in the anarchist media of inter-war Spain. International Journal of Iberian
} 
A la par, se definía de forma particular lo que debería ser la medicina y la sanidad libertaria.

En mayo de 1930, la revista mensual Mañana, editada por el grupo de inspiración sindicalista de la CNT denominado «Solidaridad», se estrenó con un artículo de Isaac Puente denominado «Medicina social ${ }^{55}$, el primero de una serie que fue vista desde Solidaridad Obrera como una "campaña social antimédica», a la que también se sumó Augusto Moisés Alcrudo desde este periódico ${ }^{56}$. Los artículos de estos dos médicos anarquistas articularon un discurso compenetrado y radical contra el sistema capitalista utilizando la medicina como base para denunciar sus relaciones sociales y proponer alternativas desde el pensamiento libertario.

Puente partía de la base de que el conocimiento médico era un patrimonio humano fruto de la experiencia acumulada, recibido como herencia de generaciones anteriores, reformado o mejorado por ellas y, por tanto, no podía considerarse propiedad exclusiva de nadie ${ }^{57}$. Este conocimiento, no se limitaba a la curación de las enfermedades sino que también era capaz de desvelar el origen social de las mismas y, consecuentemente, su profilaxis. Con estas premisas, Isaac Puente sentenciaba:

«El paro forzoso es la acción morbosa del régimen capitalista. Y el paro forzoso, para mí, "médico ante todo", es el tugurio, la indefensión contra el frío, la depauperación, el hambre crónica, la degeneración racial y el fomento de todas las enfermedades infecciosas (...) Cómo médico ante todo, debo llegar a condenar al capitalismo y a su organización social, como enemigos de la misión de la Medicina» ${ }^{58}$.

Este compromiso de los médicos, conocedores de la injusticia social, debía completarse con una labor asistencial que primara a los desfavorecidos, pues no debían consentir que «por causas ajenas a la fisiopatología» las personas encontraran diferencias en la salud y en la vida. Que unos vivieran por tenerlo todo y otros murieran por carecer de los medios vitales, sería «tanto como justificar el robo para tener salud» ${ }^{59}$. Se optaba, por tanto, por

Studies. 2011; 24 (3): 219-241.

55. Puente, Isaac. Medicina social. Mañana. 1930; 1: 7.

56. Alcrudo, Augusto M. La farsa sanitaria. Solidaridad Obrera. 4 Sep 1930.

57. Puente, n. 55.

58. Puente, Isaac. Inconformismo de un médico. La medicina ante el régimen capitalista. Solidaridad Obrera. 20 Sep 1931

59. Alcrudo, Augusto M. La Internacional de la Sanidad. Mañana. 1930; 5. 
una asistencia médica no mercantilizada al contrario de lo que era habitual dentro del sistema capitalista.

En consecuencia, otra de las líneas de actuación de los médicos anarquistas en los medios de la CNT fue la constante denuncia del entramado mercantilista que caracterizaba la relación médico-paciente bajo el sistema capitalista. Al ofrecer detalles comprometedores de los entramados ocultos de la profesión, los médicos solían utilizar un seudónimo para refugiarse de la autoridad gubernativa y de los medios no afines al anarcosindicalismo. «Un médico rural» [Isaac Puente], en un artículo titulado «El comercialismo médico», denunciaba la frecuencia con la que los médicos aplicaban técnicas diagnósticas y procedimientos terapéuticos con la única finalidad de elevar el precio de las minutas ${ }^{60}$. En marzo de 1932, el «Dr. Fantasma» [Javier Serrano Coello (1897-1974)] inauguró una serie de artículos en Solidaridad Obrera bajo el título de «Campañas sanitarias» con el propósito de desvelar los entramados ocultos de la profesión médica comenzando por los defectos formativos de las facultades de medicina ${ }^{61}$. La corrupción mercantilista de la medicina era la que provocaba el egoísmo corporativo, lo que explicaría, por otra parte, la dificultad con la que se propagaban en España los sindicatos de sanidad ${ }^{62}$. Por supuesto, el papel de los médicos funcionarios como sustentadores del Estado y defensores de la sanidad oficial tampoco escapó a las críticas de estos militantes ${ }^{63}$.

La evidente etiología social de las enfermedades fruto de la desigualdad humana y la perversión de los objetivos de la medicina legitimaban la estrategia de acción directa seguida por el anarcosindicalismo en su lucha contra el capital. De esta forma se afirmaba que no había «nada más legítimamente revolucionario que la sanidad» ${ }^{64}$. Esta legitimidad también se basaba en otro de los pilares de este pensamiento: el derecho a la salud entendido como derecho a la vida. O dicho de otra manera más acorde con el momento histórico: el derecho a no sufrir enfermedades cuyo origen social era conocido y por tanto evitables. Este derecho se extendía al de poder luchar por estos objetivos, es decir, el derecho a «luchar por la vida». Por tanto, no sólo se tendría «derecho a la salud», sino también «a

60. Un médico rural [Isaac Puente]. El comercialismo médico. Solidaridad Obrera. 28 Jul 1932.

61. Dr. Fantasma [Javier Serrano]. Campañas Sanitarias. Solidaridad Obrera. 26 Mar 1932.

62. Puente, Isaac. Los Ilamamientos a los intelectuales. Solidaridad Obrera. 6 Jul 1932.

63. Puente, Isaac. El médico funcionario. Solidaridad Obrera. 11 Mar 1931.

64. Alcrudo, n. 56. 
los medios para conservarla y a los medios para restituirnos a ella cuando el desequilibrio tenga lugar» ${ }^{65}$. Alcrudo pidió incluso la «codificación de la biología» en clave libertaria para que los médicos no pudieran actuar siguiendo su arbitrio personal porque, sentenciaba, «sobre toda clase de derechos están los metabólicos» ${ }^{66}$.

Una vez construido el derecho a la salud como el derecho más importante del trabajador, los médicos anarquistas, legitimados por sus conocimientos técnicos, ofrecieron a los obreros manuales una nueva herramienta capaz de legitimar científicamente la emancipación política y económica del proletariado. En el congreso de la federación de Sindicatos Únicos de Sanidad, podemos ver el alcance de la influencia que los profesionales de la medicina aspiraban a tener dentro de la CNT desplegando un programa de (des)medicalización absoluto sobre las masas de trabajadores, llegándose a plantear que la CNT sustituyera las tácticas de lucha económica por las de lucha sanitaria:

«Considerando como fundamental para que la sanidad sea efectiva, el asegurar a todos el derecho a la vida y a los medios y conocimientos indispensables para mantener y conservar la salud. Se consideran los ideales sanitarios equivalentes a los libertarios y se propone a la CNT la conveniencia de sustituir las tácticas de lucha económica, por las de lucha sanitaria; haciendo figurar la salud entre las reivindicaciones del proletariado» ${ }^{67}$.

Las ponencias aprobadas en este congreso incluían toda una serie de iniciativas que deberían ser llevadas por Comités de Defensa Sanitaria locales, que se ocuparían de desarrollarlas en el seno de la CNT. Entre ellas podemos destacar la propaganda e instrucción sanitaria, la elaboración de un censo de productores para la planificación de la futura sanidad o la fiscalización de las condiciones higiénico-sanitarias del trabajo.

De acuerdo con las relaciones de inclusión-exclusión que estamos analizando, condicionadas también por las prioridades sindicales que a

65. Alcrudo, Augusto M. Sanidad de vanguardia. El derecho a la salud. Mañana. 1931; 8.

66. Alcrudo, n. 59. Sobre el concepto de salud en clave anarquista, véase: Molero-Mesa, JiménezLucena, n. 54; Jiménez-Lucena, Isabel; Molero-Mesa, Jorge. Problematizando el proceso de (des)medicalización. Mecanismos de sometimiento/autogestión del cuerpo en los medios libertarios españoles del primer tercio del siglo XX. In: Miranda, M.; Girón Sierra, A. eds. Cuerpo, biopolítica y control social: América Latina y Europa en los siglos XIX y XX. Buenos Aires: Siglo XXI; 2009, p. 69-93.

67. Congreso de Sindicatos Únicos de Sanidad. Solidaridad Obrera. 22 Nov 1931. 
su vez quedaban limitadas por las fuertes medidas represivas que irían estableciendo progresivamente los distintos gobiernos republicanos contra el movimiento libertario, podemos entender que ninguna de estas propuestas fueran discutidas en los sindicatos locales ni tenidas en cuenta en los distintos congresos de la CNT que se realizaron en este periodo. No eran prioritarias. Ahora bien, las derivaciones del análisis libertario a las relaciones médico-enfermo afectaban por completo a la forma en la que se establecía la asistencia sanitaria bajo el sistema capitalista. La beneficencia, las mutuas comerciales, o incluso los seguros obligatorios estatales, como el de maternidad, eran vistos como elementos sustentadores de la desigualdad social y obstáculos para alcanzar la revolución ${ }^{68}$.

De esta forma, las denuncias contra las mutuas de enfermedad, constantes en las páginas de la prensa anarquista ${ }^{69}$, cobraron un fundamento científico de la mano de los médicos anarquistas, estableciéndose otra vía de inclusión a través de un tema especialmente sensible para los obreros, como eran las consecuencias de los accidentes del trabajo. Junto al abuso de autoridad de las mutuas, la humillación del trabajador que acude a reclamar sus derechos y la negativa a prestar asistencia a los mutualistas atendiendo a la letra pequeña, el médico era acusado de actuar en contra de los intereses de los trabajadores, de sacrificar «la nobleza de su profesión»y de convertirse en «un burócrata de ciencia sin conciencia» ${ }^{70}$. Javier Serrano, desde su sección en Solidaridad Obrera denominada «Folletín sanitario» anunció que las mutuas habían hecho «un descubrimiento maravilloso»: la hernia abdominal no se producía porque el obrero sufriera un golpe en un accidente laboral, sino porque «el obrero tiene las paredes abdominales flojas». La lógica con la que actuaba el sistema burgués incluía al resto de las relaciones sociales en las que, él mismo, se incluía como afectado:

«Cualquier día uno de esos autos burgueses que corren velozmente nos aplastará con su masa y el opulento burgués que lo guíe nos pedirá daños y perjuicios por haberle salpicado las ruedas con nuestra sangre ${ }^{71}$.

\footnotetext{
68. Jiménez Lucena, n. 23.

69. Jiménez Lucena, Isabel; Molero Mesa, Jorge. Per una «sanitat proletària». L'Organització Sanitària Obrera de la Confederació Nacional del Treball a la Barcelona republicana, (1935-1936). Gimbernat. 2003; 39: 211-221.

70. Notas del día. Solidaridad Obrera. 2 Jul 1931.

71. Serrano, Javier. Folletín sanitario. Las mutuas de seguros. Solidaridad Obrera. 17 Feb 1933.
} 
La complicidad de la justicia oficial con las mutuas también fue denunciada por Serrano que reconoció haber actuado como perito en varios pleitos planteados por obreros y haberlos perdido todos a causa de la legislación vigente y la actitud de los jueces.

La estrategia a seguir para hacer frente a este tipo de problemas derivados de la desigualdad humana en la sociedad capitalista dividió al sindicalismo a comienzos del siglo XX entre aquellos que veían factible la cooperación «en los ramos de consumos, socorros e instrucción», el llamado «sindicalismo a base múltiple», y los que, por el contrario, rechazaban esta posibilidad «reformista»y se decantaron por la «acción directa frente al capital». La CNT, como ya hemos visto, optó por esta última en su congreso fundacional de 1910, lo que significaba no realizar ninguna actividad dentro de los sindicatos que los apartaran de sus objetivos revolucionarios a corto plazo. Renunciaron incluso a las «cajas de resistencia» para el mantenimiento de los obreros en huelga, convencidos de que la acumulación de dinero en los sindicatos nunca superaría al que poseía el capital ${ }^{72}$. Los miembros de la Confederación, por tanto, debían solventar por su cuenta la pertenencia a sociedades asistenciales ya fuesen de autoayuda o comerciales, pero siempre fuera de la CNT.

Esta estrategia sindical, unida a las consecuencias antes vistas del apoliticismo libertario, impidió que los médicos establecieran cualquier tipo de interdependencia comercial y política con los sindicatos anarcosindicalistas. En este sentido, los médicos anarquistas que comulgaban con la acción directa, contribuyeron además a evitar que los sectores reformistas de la CNT completasen, en 1931, la creación de una mutua dedicada a la curación de la tuberculosis denominada Obra Popular Antituberculosa de Cataluña ${ }^{73}$. El papel de los médicos militantes, como Isaac Puente, fue fundamental en el fracaso de la mutua ${ }^{74}$ que, apoyada por los máximos responsables de la cúpula sindical y de la dirección de Solidaridad Obrera ${ }^{75}$, quedó descartada,

72. Este rasgo le separaba radicalmente de la UGT que en 1904, había creado «La Mutualidad Obrera Cooperativa médico-farmacéutica y de enterramientos de trabajadores asociados». Este sindicato socialista asumió oficialmente el sindicalismo a base múltiple en su congreso de 1907. Castillo, Santiago. El socialismo madrileño hace un siglo: Un anhelo de reformas. Arbor. 2001; 159 (666): 411-429.

73. Farreras. Una iniciativa. La tuberculosis y el obrero I. Solidaridad Obrera. 26 Feb 1931.

74. Puente, Isaac. Ante una iniciativa. Inquietudes proletarias. Solidaridad Obrera. 6 Mar 1931.

75. El proyecto estaba avalado por una parte importante de la cúpula dirigente del sindicato comenzando por Ángel Pestaña y varios miembros de la redacción de Solidaridad Obrera, 
después de ocho meses de campaña, en el Pleno de Sindicatos locales de Badalona en octubre de $1931^{76}$. El desgaste sufrido por el sector reformista en su apoyo a la campaña contribuyó de manera importante a la escisión de la CNT pocos meses después ${ }^{77}$.

La respuesta de los médicos militantes, una vez ratificada la estrategia de acción directa a nivel mutual, fue utilizar sus servicios asistenciales de manera desinteresada con los obreros de la CNT a través de consultas médicas gratuitas. En efecto, como prueba irrefutable de su adhesión al movimiento anarcosindicalista, en el que ya colaboraban puntualmente con dinero para sostener la prensa o el apoyo a los presos, ofrecieron sus consultas particulares para atender gratuitamente a los enfermos de los sindicatos que estuvieran sin trabajo o hubiesen sido agraviados por las compañías de seguros. Contando con algunas experiencias previas y malogradas de los extintos Sindicatos de sanidad ${ }^{78}$, Alfredo Royo y Javier Serrano comenzaron a ofrecer esta posibilidad a través de Solidaridad Obrera en octubre de 1932. La acogida fue muy buena por parte de los confederados, hasta el punto de que la redacción de Solidaridad Obrera pidió que la iniciativa sirviese de estímulo al resto de intelectuales sindicados para que actuaran de igual manera ${ }^{79}$. El «rasgo humanitario» de Serrano también fue alabado por Solidaridad Obrera en contraposición a aquellos médicos que por mercantilismo, habían convertido su profesión en «algo denigrante» ${ }^{80}$. El anarcosindicalismo, por primera vez, vio una oportunidad en conseguir esa «unión del brazo y del cerebro» no sólo con los médicos militantes sino también con el resto de los técnicos sanitarios, ya fuesen afiliados o meros simpatizantes.

A lo largo de varios años, en los que la afiliación a la CNT fue motivo de persecución por parte de los gobiernos republicanos y en los que fracasaron distintos movimientos revolucionarios, podemos ver a través de la prensa

encabezados por su director, Juan Peiró (1887-1942) y el redactor Ramón Magré (n. 1899). Una obra humanitaria. Solidaridad Obrera. 30 Abr 1931. Aprobados los estatutos. La Vanguardia. 26 Ago 1931.

76. Farreras Munner, Dr. La tuberculosis y el obrero II. Solidaridad Obrera. 10 Ago 1932.

77. En noviembre de 1931 se creó en Madrid una «Mutua Obrero-sanitaria». Sindicato Único de Sanidad de Madrid. El Sol. 3 Nov 1931. Pero no nos consta que llegara a funcionar después de las depuraciones de los militantes del Sindicato de Sanidad.

78. El Sindicato de Sanidad. Solidaridad Obrera. 9 Oct 1931.

79. Importante. Solidaridad Obrera. 16 Oct 1932.

80. Rasgo humanitario. Solidaridad Obrera. 29 Oct 1932. 
anarcosindicalista una evolución positiva y ascendente de esta estratégica relación entre los médicos y los obreros que culminó con la creación, en mayo de 1935, de la Organización Sanitaria Obrera (en adelante OSO). Esta organización tuvo una evolución muy significativa en sus objetivos. Comenzó organizando una serie de consultorios gratuitos para los obreros sin recursos y acabó convertida en una organización mutual con planteamientos muy ambiciosos ${ }^{81}$. Sin embargo, la realidad de la OSO fue que el dinero que se recaudaba en suscripciones de los sindicatos, venta de sellos y otras actividades, se consumía de inmediato ya que, a pesar de que los médicos no cobraban por las consultas, los medicamentos eran dispensados gratuitamente, lo que no dejaba margen de mejora ni tampoco para levantar un proyecto que surgió de los médicos de la OSO: la construcción de un hospital proletario. La necesidad de dinero terminó convirtiendo a la OSO en una mutua de pago muy especial pero que, para algunos militantes, contradecía el principio de la acción directa. Esta derivación hay que entenderla por las nuevas circunstancias en las que se encontraba la CNT después del desgaste que supusieron los distintos fracasos revolucionarios, el auge del fascismo y la presión para formar el frente único de trabajadores con la UGT. La reunificación de los sectores anarcosindicalistas en 1936 y la creencia en la inminente revolución social, llevó a convertir a la OSO en el germen de una posible organización sanitaria sustitutiva de la sanidad burguesa ${ }^{82}$.

\section{Epílogo}

El estallido de la Guerra civil cambió por completo el panorama en el que se estaban desarrollando las estrategias de inclusión-exclusión que hemos analizado en el presente trabajo. De forma inmediata, se reactivaron o se organizaron de nuevo los sindicatos únicos de sanidad en varias provincias de la zona republicana, ahora bajo el impulso de una futura «colectivización de la medicina». El nuevo Sindicato Único de Sanidad de Barcelona se constituyó el 16 de agosto de $1936^{83}$ cuando la Consejería de Sanidad y Asistencia Social de la Generalitat ya había comenzado el proceso de

81. Jiménez Lucena, Molero Mesa, n. 69.

82. Jiménez Lucena, Molero Mesa, n. 69; Jiménez Lucena, n. 23.

83. Sindicato Único de Sanidad. Solidaridad Obrera, 16 Ago 1936: 15. 
incautación de los centros sanitarios de la Iglesia y de las mutuas sanitarias privadas. De forma paralela el Comité Sanitario de Milicias Antifascistas ya controlaba las organizaciones médicas que se concentraban en el Casal del Metge: Sindicat de Metges de Catalunya y su Mutual Mèdica de Catalunya i Balears, el Col.legi de Metges de Barcelona y la Acadèmia de Ciències Mèdiques de Catalunya i Balears. El resultado de todos estos acontecimientos fue el ingreso masivo de médicos y de profesionales de todas las ramas sanitarias en el Sindicato Único de Sanidad ${ }^{84}$. La organización sanitaria anarcosindicalista culminó en marzo de 1937 con la creación de la Federación Nacional de Sindicatos Únicos de Sanidad dentro del Primer Congreso Nacional de Sanidad celebrado en Valencia. En este congreso, la ponencia final de la sección de Practicantes afirmaba que «todas las luchas intestinas entre los profesionales sanitarios, hasta aquí existentes, desaparecen toda vez que el Congreso ha establecido dos principios revolucionarios: la socialización de la sanidad y el libre acceso a la enseñanza sin más exigencias que la capacitación ${ }^{85}$. Comenzaba una nueva etapa que será el objeto de nuestras próximas investigaciones.

84. Hervas Puyal, Carlos. Sanitat a Catalunya durant la República i la Guerra Civil. Barcelona, Universitat Pompeu Fabra; 2005.

85. Memorias del Primer Congreso Nacional de Sanidad, CNT-AIT. Valencia: Ed. Guerri colectivizada; 1937, p. 44. 


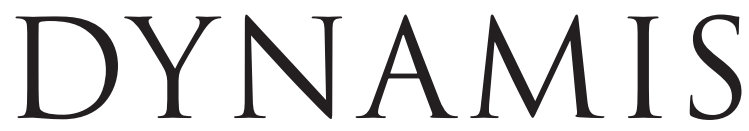

Acta Hispanica ad Medicinae Scientiarumque Historiam Illustrandam VOLUME 33 (1)

«Arm and Brain»: Inclusion-exclusion dynamics related to medical professionals within Spanish anarcho-syndicalism in the first third of the 20th century

Jorge Molero-Mesa and Isabel Jiménez-Lucena....................

1.--Inclusion and exclusion of technicians and intellectuals in anarchosyndicalism. 2.-The Single Health Unions and the National Confederation of Labour. 3.- Inclusion-exclusion of physicians in anarcho-syndicalism. Strategies, resistances and limitations. 4.-Epilogue.

ABSTRACT: The aim of this paper is to analyze the dynamics within Spanish anarcho-syndicalism between manual workers and medical professionals who shared the anarchist ideology. The incorporation of technicians into the labour movement was a common feature in the Western world; however, while socialist organizations left health policies in the hands of physicians, anarchist 
unions did not accept these technocratic principles, given that they did not consider intellectuals as the best suited to take decisions concerning the whole community. In this context, we can see how medical professionals developed diverse strategies to be accepted by anarcho-syndicalist militants, who in turn showed different levels of acceptance according to the distinct lines of thought within the union. 